\title{
O PAPEL DAS COOPERATIVAS AGROPECUÁRIAS: PROCESSO DE CAPACIDADE ABSORTIVA DE PRODUTORES RURAIS ASSOCIADOS
}

\author{
The role of agricultural cooperatives in the absortive capacity process \\ of associated rural producers
}

\begin{abstract}
RESUMO
Nosso estudo objetivou compreender como ocorre o processo da capacidade absortiva (CA) em firmas de produtores rurais associadas a cooperativas. Trata-se de um estudo de caso, realizado com seis produtores rurais de uma cooperativa do setor agropecuário do Estado de Santa Catarina. A coleta de dados ocorreu por meio de entrevistas em profundidade. Para analisar os dados recorreu-se à análise das conteúdo a partir das quatro dimensões da CA, propostas por Zahra e George (2002). Os dados evidenciaram que os produtores rurais buscam conhecimento de forma autônoma e via cooperativa. Quanto a assimilação, os produtores rurais assimilam as informações externas a fim de adaptá-las e aplicá-las em suas propriedades tanto de forma autônoma como com o auxílio da cooperativa. No processo de transformação do conhecimento identificamos que os produtores possuem o hábito de discutir as tendências do mercado com as empresas parceiras da cooperativa, com o departamento técnico e no comitê tecnológico que foi criado para discutir tendências. No que tange a categoria exploração do conhecimento, foi constatado que os entrevistados perseguem o uso da tecnologia, notadamente o uso de softwares e equipamentos. Argumentam que as informações geradas pelos softwares auxiliam na tomada de decisão, pois possibilitam analisar o desempenho da área plantada, otimizando os recursos, com o intuito de minimizar os custos, melhorando a produtividade e aumentando a rentabilidade. Finalmente nosso estudo mostra que o conhecimento absorvido via cooperativa é mais rapidamente assimilado e mais bem explorado, facilitando adoção de inovações.
\end{abstract}

Camila Camargo Aguiar

Universidade do Oeste de Santa Catarina

professoracamilaadm@hotmail.com

Silvio Santos Junior

Universidade do Oeste de Santa Catarina

silviosantos.junior@unoesc.edu.br

Juliano Danilo Spuldaro

Universidade do Oeste de Santa Catarina

juliano.spuldaro@unoesc.edu.br

Recebido em: 21/08/2020. Aprovado em: 14/05/2021.

Avaliado pelo sistema double blind review

Avaliador científico: Eduardo Cesar Silva

DOI: $10.48142 / 2320211690$

\begin{abstract}
Our study aimed to understand how the absorptive capacity (AC) process occurs in rural producer firms associated with cooperatives. This is a case study, carried out with six rural producers from a cooperative in the agricultural sector in the State of Santa Catarina. Data collection took place through in-depth interviews. To analyze the data, content analysis was used from the four dimensions of AC, proposed by Zahra and George (2002). The data showed that rural producers seek knowledge autonomously and via cooperatives. As for assimilation, rural producers assimilate external information in order to adapt and apply it to their properties both autonomously and with the help of the cooperative. In the knowledge transformation process, we identified that producers have the habit of discussing market trends with the cooperative's partner companies, with the technical department and in the technological committee that was created to discuss trends. Regarding the knowledge exploration category, it was found that respondents pursue the use of technology, especially the use of software and equipment. They argue that the information generated by the software helps in decision making, as they make it possible to analyze the performance of the planted area, optimizing resources, with the aim of minimizing costs, improving productivity and increasing profitability. Finally, our study shows that knowledge absorbed via cooperatives is more quickly assimilated and better exploited, facilitating the adoption of innovations.
\end{abstract}

Palavras-chave: Capacidades Dinâmicas; Cooperação; Inovação tecnológica.

Key words: Dynamic Capabilities; Cooperation; Technological Innovation. 


\section{INTRODUÇÃO}

O setor do agronegócio é parte fundamental do desenvolvimento e crescimento do Brasil (Barcellos, Lampert, Grundling \& Canellas, 2010). De acordo com a Confederação da Agricultura e Pecuária do Brasil (CNA, 2020), o agronegócio contribui de forma significativa para a balança comercial. Projeções do Ministério da Agricultura, Pecuária e Abastecimento (MAPA, 2013, p. 3) indicam que "o agronegócio brasileiro caminha para a próxima década com foco na competitividade e na modernidade, fazendo da utilização permanente da tecnologia um caminho para a sustentabilidade". Furlan, Angnes e Morozini (2018) corroboram que a utilização de tecnologias e inovações no campo aumenta a produtividade e resulta em melhor desempenho nas propriedades rurais.

Para melhor competir nesta realidade dinâmica, Cohen e Levinthal (1990) argumentam para necessidade de as firmas desenvolverem Capacidade Absortiva (CA), entendida como a habilidade coletiva da firma em reconhecer o valor de um novo conhecimento externo, assimilá-lo e aplicá-lo a fins comerciais. De forma complementar, Zahra e George (2002, p.186) argumentam que a CA representa "um conjunto de rotinas e processos organizacionais por meio dos quais as empresas adquirem, assimilam, transformam e aplicam o conhecimento com propósito de produzir uma capacidade dinâmica e sustentável." Assim sendo, a CA pode propiciar o conhecimento para as práticas inovadoras em propriedades rurais (Vermeire, Viaene \& Gellynck, 2009), e, de modo consequente, a melhora no desempenho de firmas do agronegócio.

Nessa linha de raciocínio, as cooperativas podem ser entendidas com estruturas que possibilitam economizar custos de transação, especializar tarefas e garantir um controle de qualidade dos insumos necessários para a atividade da agricultura (Valentinov, 2007). Deste modo, concordando com Binotto, Siqueira e Nakayama (2009), a forma organizacional cooperativa favorece a $\mathrm{CA}$ ao permitir que os agentes envolvidos se aproveitem também da similaridade de experiências, processos e objetivos durante o processo.

No entanto, ainda são poucos os estudos que discutem a capacidade absortiva em cooperativas e no agronegócio. Helfat e Peteraf (2009) ressaltam que as capacidades dinâmicas podem ser aplicadas em diversas configurações, em empresas de qualquer porte ou segmento. Contudo, Wilson e Kevill (2017) evidenciam a escassez de pesquisas sobre a aplicação das Capacidades Dinâmicas em pequenas e microempresas, principalmente no segmento rural. Corroborando, Szintowski (2017, p.21, tradução nossa) argumenta que "embora a CA tenha sido estudada em diferentes contextos e níveis [...], ainda há lacunas a serem preenchidas com tal abordagem e, como exemplo, cita-se o agronegócio".

Entendemos, pois, que há necessidade de avançar em pesquisas e evidências, que possam explicar melhor como ocorre o processo de CA em cooperativas agropecuárias. Assim, a questão norteadora do nosso estudo será: Como as cooperativas agropecuárias influenciam na $\mathrm{CA}$ de produtores rurais associados? Analisamos as dimensões do processo da capacidade absortiva de acordo com o modelo de Zahra e George (2002), tendo em vista sua relevância, aceitação e abrangência. Sendo assim, o objetivo deste estudo é compreender como as cooperativas influenciam o processo de capacidade absortiva de produtores rurais associados.

Os resultados que encontramos mostram que os produtores procuram acompanhar as mudanças do setor, e que não se limitam a fazer isso por meio da cooperativa, embora destaquem que ela exerce papel fundamental. $\mathrm{Na}$ análise de conteúdo por co-ocorrência realizada foi possível avaliar que a cooperativa serve como um ente mediador que agiliza e facilita a absorção de conhecimentos. A transformação do conhecimento, com apoio do departamento técnico da cooperativa, é facilitada. O estudo indica também que os produtores entendem a exploração do conhecimento na propriedade rural como sendo concretizada por meio do uso da tecnologia, ou seja, através do uso de softwares e equipamentos. Nessa linha, discutimos que há uma promoção da adoção de inovações tecnológicas.

Estruturalmente o estudo encontra-se dividido, além da introdução, em mais cinco seções. A segunda seção apresenta um breve resgate sobre os tópicos norteadores do estudo. A terceira seção traz detalhes do método utilizado e do campo pesquisado. A quarta seção apresenta a descrição e análise dos dados coletados, a quinta seção traz uma breve análise com vistas às considerações finais, seguidas pelas referências.

\section{REFERENCIAL TEÓRICO}

Desde que o pêndulo da explicação do desempenho superior e da vantagem competitiva pendeu a última vez para dentro das firmas (Hoskisson, Wan, Yiu \& Hitt, 1999), passou-se a considerar que as capacidades organizacionais (Chandler, 1992; Dosi, Nelson \& Winter, 2000) são elementos fundamentais do seu funcionamento. A partir de então, muitas capacidades foram mapeadas e buscou-se compreender seu impacto no desempenho das firmas (Ferreira \& 
Fernandes, 2017). Entre elas, estão duas capacidades com papéis especiais: as capacidades dinâmicas (Teece, Pisano \& Shuen, 1997), responsáveis por articular e modificar outras capacidades, rotinas e recursos e as capacidades absortivas (Cohen \& Levinthal, 1990), mais recentemente compreendidas como um importante elemento do processo estratégico organizacional, que estimula a gestão do conhecimento, a aprendizagem e a inovação dentro das firmas.

A literatura alega que as capacidades absortivas sejam compostas de fases (Zahra \& George, 2002). Porém, não há consenso sobre quais sejam estas fases, visto que vários modelos têm sido apresentados e discutidos (Lane, Koka \& Pathak, 2006; Todorova \& Durisin, 2007). Apesar dos avanços, também não se tem uma visão definitiva sobre os antecedentes e os resultados (Jansen, Van Den Bosch \& Volberda, 2005; Rezaei-Zadeh \& Darwish, 2016) que a influenciam e que ela produz. Assim, com as definições clássicas já claras, com modelos disponíveis e interessados em antecedentes e resultados, a pesquisa sobre capacidade absortiva tem experimentado verificar sua existência em contextos diversos.

O modo com que o processo de capacidade absortiva produz resultados (outcomes) depende fundamentalmente das características do contexto organizacional em que isso ocorre (Lane et al., 2006). As cooperativas do setor agropecuário representam terreno fértil para a análise da capacidade absortiva e seus aspectos cognitivos (Fontanari, 2017). Para compreender as influências das características organizacionais específicas do modelo de cooperativas na capacidade absortiva, entretanto, é necessário compreendê -la genericamente em primeiro lugar. Na sequência deste referencial o conceito é revisado para então ser debatido no contexto organizacional (cooperativas) e setorial (agropecuária) de estudo.

\subsection{O processo de capacidade absortiva}

Considerada um constructo multidimensional, a capacidade de absorção é tida como a capacidade da empresa em "reconhecer o valor da informação nova, externa, assimilá-la e aplicá-la para fins comerciais [...] avaliar e utilizar o conhecimento exterior, em grande parte em função do nível de conhecimento prévio". de Cohen e Levinthal (1990, p.128). Para Zahra e George (2002), a capacidade absortiva é composta por quatro dimensões: aquisição; assimilação; transformação e exploração do conhecimento.

A aquisição se refere à capacidade da empresa em identificar e adquirir conhecimento gerado fora da organização. A identificação do conhecimento de forma bem operada pode estabelecer melhor a qualidade de aquisições da empresa (Zahra \& George, 2002; Kim, 1997). Já a assimilação do conhecimento está relacionada com a capacidade de compreensão no sentido de aprender com as novas informações adquiridas de modo que elas influenciem positivamente rotinas e processos organizacionais (Kim, 1997; Szulanski, 1996). Segundo Tepic, Trienekens, Hoste e Omta (2012) a capacidade de assimilação, por sua vez, contribui de modo crucial para os aspectos de inovação, como por exemplo adoção de inovações ou inovação de processo. Já a dimensão de transformação do conhecimento expressa a capacidade da empresa para desenvolver e melhorar as rotinas que facilitam a combinação do conhecimento existente com o conhecimento adquirido e assimilado visto que a habilidade de transformar novos conhecimentos está diretamente relacionada à capacidade de renovação da empresa (Christensen, Suarez \& Utterback, 1998). Por fim, a dimensão de exploração reflete a capacidade da empresa para incorporar o conhecimento adquirido nas suas operações (Tiemessen, Lane, Crossan, \& Inkpen, 1997; Van den Bosch, Volberda \& Boer, 1999) resultando em novos produtos, sistemas, processos e até mesmo novas formas organizacionais (Spender, 1996).

Posteriormente, o entendimento do conceito evoluiu com a contribuição de Lane et al. (2006) para uma definição mais processual e compartimentada da ideia de capacidade, por meio de três processos sequenciais. Os autores mencionam que é necessário reconhecer e, ao mesmo tempo, entender os conhecimentos que são potencialmente novos e valiosos, que estão fora da firma através da aprendizagem exploratória. Depois é preciso assimilar o novo conhecimento considerado valioso por meio da aprendizagem transformativa. Por fim, a firma deve apropriar-se do conhecimento assimilado visando criar novos conhecimentos e resultados comerciais por meio de aprendizagem proveitosa.

Dessa forma, o conhecimento, seja ele ainda no âmbito externo, no interno ou já transformado, assume papel fundamental na constituição de uma capacidade (Bingham, Eisenhardt \& Furr, 2007). Acima disso, o conhecimento é um recurso importante para as organizações (Grant, 1996), pois é fundamental para o desempenho (Vasconcelos \& Cyrino, 2000) possibilitando o alcance de posições de vantagem competitiva. As organizações têm reconhecido que o conhecimento tem se transformado em um recurso necessário para mantê-las competitivas e bem-sucedidas em longo prazo (Luchesi, 2012) por meio da criação e adoção de inovações. Para Karkoulin, Harake e Messarra (2010) compartilhar o conhecimento dentro do contexto organizacional implica em resultados positivos como melhor produtividade, aprendizado e inovação. Nesse 
sentido Lane et al., (2006) mencionam que para a sobrevivência sustentada de uma organização, é fundamental para o desenvolvimento e a manutenção da capacidade de absorção, no sentido de reforçar e reorientar a base de conhecimentos da organização. Compreende-se que a capacidade absortiva se relaciona com a inovação quando os processos de aquisição, assimilação, transformação e aplicação do conhecimento são elementos promotores da inovação das empresas, seja ela de produto, processo, organizacional ou mesmo a adoção de inovações.

\subsection{Cooperativas como uma configuração organi- zacional típica do setor agropecuário}

Desde Cook (1995) questiona-se a sustentabilidade e eficiência do modelo de cooperativas. Sabe-se que significativas mudanças nas formas organizacionais adeptas ao modelo cooperativo de agricultura têm ocorrido ultimamente (Fulton \& Hueth, 2009) e é evidente que o benefício social do cooperado já não é tão relevante quanto fora antigamente (Nilsson, Svendsen \& Svendsen,2012). Ainda assim, os cooperados que integram este tipo de forma organizacional se beneficiam de diversas formas, mesmo que marginalmente no cenário atual. Enquanto alguns estudos enfatizam aspectos menos tangíveis do benefício ao cooperado como confiança, reputação e capital social (Nilsson et al., 2012) outros enfatizam as economias decorrentes dos custos de transação, especialização das tarefas (Valentinov, 2007) e a capacidade de coordenação (Sporleder, 1992). No Brasil - um país tipicamente propício para a atividade agropecuária - por exemplo, as cooperativas adotaram uma estratégia de internacionalização e diversificação de seus produtos visando manter-se competitivas e atrativas à associados (Ritossa \& Bulgacov, 2009).

Mais recentemente, suscitou-se que pode haver uma dimensão de conhecimento que ajuda a sustentar este tipo de arranjo organizacional (Fontanari, 2017). Nesse sentido, a estrutura organizacional cooperativa pode auxiliar os associados a obterem benefícios no sentido de acessar, assimilar e aproveitar conhecimento que esteja localizado no exterior da organização, inovando ou adotando inovações (Vespo, 2013). Diferentemente do que aconteceu, por exemplo, com as pressões ambientais para adoção de práticas orgânicas na agricultura cooperativa (Shanahan, Hooker \& Sporleder, 2008), no caso do conhecimento não há um processo isonômico de incorporação. Cada organização, mesmo que associada, está sujeita ao seu conhecimento específico ao adotar ou criar inovações decorrentes do conhecimento absorvido.

\subsection{Capacidade absortiva e cooperativas}

Vermeire et al. (2009), ressaltam que a capacidade absortiva tem sido relevante para a transmissão de conhecimento e para adoção de práticas inovadoras em propriedades rurais. A capacidade absortiva tem origem nos estudos de Cohen e Levinthal (1989), que destacam a importância da aprendizagem organizacional, que envolve a identificação, a assimilação e a aplicação de conhecimentos do ambiente externo para fins comerciais, a partir da pesquisa e desenvolvimento e dos aspectos cognitivos referentes ao processo de aprendizagem. Assim, a capacidade absortiva é importante para a inovação em setores tradicionais, bem como no setor rural (Gellynck et al., 2015).

A capacidade de inovação é um dos aspectos mais relevantes do processo empresarial e reflete na capacidade da empresa em participar e apoiar novas ideias, processos criativos e experimentação, que consequentemente podem resultar em novos produtos ou até mesmo processos tecnológicos (Lumpkin \& Dess1996; Rhee, Park \& Lee, 2010).

As empresas tendem a convergir os seus conhecimentos transformando-os em capacidades no sentido de atender as exigências do mercado e do setor onde estão inseridas (Lane \& Lubatkin,1998). De maneira especial o conhecimento compartilhado é relevante dentro das organizações cooperativas, que se constituem com a finalidade de trabalhar solidariamente. Dessa forma, o compartilhamento do conhecimento e a capacidade absortiva se constituem em grandes desafios para a gestão das organizações rurais, notadamente as estruturas organizacionais cooperativas.

Nesse sentido as empresas rurais (produtores rurais) buscam a inovação por meio do conhecimento técnico ou do conhecimento científico (Balestrin \& Verschoore, 2010) como forma de se manterem competitivos na economia atual. Estudo de Binotto et al. (2009) evidenciam, entretanto, que a que a propriedade rural tem mais dificuldade em gerar conhecimento de forma isolada, pois necessidade dedicar ativos específicos comprometidos nas atividades na busca pela inovação. Assim, as cooperativas podem ser consideradas como o "mecanismo de interação social" - nos termos de Zahra \& George (2002) - necessário para facilitar o desenvolvimento da capacidade absortiva da empresa rural.

Corroborando, a pesquisa realizada por Tepic et al. (2012) com produtores de suínos nos países baixos, constatou que a interação com diferentes atores é relevante para acumular informação e consequentemente conhecimento no que tange aos aspectos de inovação. O estudo encontrou relação positiva entre aquisição de conhecimento e capacidade de estabelecer contatos com parceiros que 
forneçam informações setoriais (tendências, regulamentos e técnicas). Tanto os estudo de Tepic et al.(2012) quanto de Binotto et al. (2009) mostram que a frequência de interação dos produtores influencia na capacidade absortiva.

No estudo de Binotto et al. (2009) realizado em dois países, constatou os produtores rurais no Brasil estão inseridos num contexto cooperativo e, na Austrália, os negócios são conduzidos individualmente, mas observam que "para a ocorrência da criação de conhecimento, é necessária a existência de grupos de relacionamento e de certa liderança em nível de propriedade, de comunidades de prática ${ }^{1}$ ou da cooperativa" (p.1). Também devido ao suporte da cooperativa, há tendência de redução nos riscos no gerenciamento da atividade dos produtores associados. Ou autores ainda concluíram que o processo de criação de conhecimento está institucionalizado na cooperativa por meio das diversas formas de interação com os associados e dos vínculos existentes entre as partes. Já na Austrália, os produtores demonstraram não possuir uma estrutura de suporte como a dos brasileiros, os mesmos percebem a necessidade de aprendizagem permanente para se autodesenvolver. Sendo assim, fica evidente o papel relevante das cooperativas Brasileiras como ambiente propícios para incentivar a capacidade absortiva, novos conhecimentos e novas práticas na propriedade rural.

Reagans e McEvily (2003) do mesmo modo evidenciaram que uma rede pode ser importante para ganhar conhecimento externo, ou seja, um indivíduo como ampla rede de contatos e experiências pode preencher espaços a cerca de conhecimentos diversificados. Em relação as novas práticas na propriedade rural, destaca-se o papel da tecnologia, que segundo Alves (2012) resulta na redução nos custos de produção, na melhora da produtividade, e de forma consequente tornando a propriedade mais lucrativa, eficiente e competitiva. A tecnologia para Gelinski Neto (2012) possui um caráter microeconômico de aumentar a produtividade e reduzir o custo médio na propriedade, dessa forma melhorando a rentabilidade do produtor

De acordo com Buainain, Alves, Siqueira e Navarro (2014), a agricultura está entre os setores mais impactados pela tecnologia. Os autores ressaltam que a maior parte das atividades de pesquisas realizadas no país estão preocupadas com aspectos referentes a novos processos de

${ }^{1}$ Para os autores as comunidades práticas "não se constituem num processo tecnológico, e sim num processo social e cognitivo. Sua formação não depende somente de tecnologia, mas do estabelecimento de relações informais, nas quais as pessoas podem utilizar esses ambientes para realizar trocas, definir suas verdades quanto às suas posturas frente às mudanças" Binotto et al. (2009, p.1). produção, com o desenvolvimento de novos produtos e o melhoramento da genética. Gelinski Neto (2012) ainda afirma que a tecnologia para a agricultura possui caráter sistêmico e envolve uma interação cada vez maior entre os produtores e o mercado.

\section{MÉTODO}

Com o objetivo de compreender como as cooperativas agropecuárias influenciam o processo de capacidade absortiva de produtores rurais associados, realizamos um estudo com seis (6) produtores de uma cooperativa. O lócus de pesquisa foi a cooperativa Copercampos ${ }^{\circledR}$ na cidade de Campos Novos - SC. A escolha se justifica devido a Copercamos ${ }^{\circledR}$, ter a maior produtividade no meio oeste catarinense. Vale destacar ainda que, em 2019 ocupava a posição de segunda maior produtora de sementes de soja do país. Produziu 64 mil toneladas de sementes, com um faturamento superior a R $\$ 238$ milhões. A alta tecnologia utilizada no campo, segundo Relatório Anual (2019) é a razão deste desempenho. Assim a Copercampos ${ }^{\circledR}$ pareceu o ambiente apropriado nosso estudo.

Como sujeitos da pesquisa, contamos com a participação de seis (6) membros associados da cooperativa, que são dirigentes de empresas rurais, e foram escolhidos de maneira intencional, e por conveniência. Portanto, para compor o grupo de entrevistados, definiu-se alguns critérios como: tamanho da firma associada em termos de área cultivada (não menor que quatro módulos rurais); tempo de associado (não menos de 10 anos); quantidade de cultivares de plantio na propriedade; e utilização de soluções tecnológicas, notadamente em equipamentos com tecnologia embarcada (uso de softwares, equipamentos agrícolas modernos, aplicativos entre outros). Os sujeitos escolhidos, e algumas características de suas firmas, são descritos no Quadro 1.

Como coleta de dados utilizou-se de entrevistas semiestruturadas com categorias de análise definidas a priori, aplicadas aos associados da Copercampos ${ }^{\circledR}$. As entrevistas foram divididas em dois momentos, no primeiro momento procurou compreender características gerais das firmas associadas como escolaridade, idade, número de funcionários, área cultivada, bem como as culturas desenvolvidas na propriedade. Já no segundo momento abordou questões referentes ao processo de capacidade absortiva de acordo com o modelo proposto por Zahra e George (2002) sendo elas: 1. Aquisição do conhecimento, 2. Assimilação do conhecimento, 3. Transformação do conhecimento e 4. Exploração do conhecimento. 
QUADRO 1 - Descrição dos sujeitos da pesquisa

\begin{tabular}{|lcccccc|}
\hline $\begin{array}{c}\text { Cód. da firma } \\
\text { pesquisada }\end{array}$ & Idade & Gênero & Escolaridade/ profissão & $\begin{array}{c}\text { N. de } \\
\text { funcionários }\end{array}$ & Área cultivada & Culturas cultivadas \\
\hline Produtor 1 (P1) & 48 anos & Masc. & Ensino médio & 05 & 1.450 hectares & $\begin{array}{c}\text { Soja, milho, feijão, trigo e } \\
\text { cevada }\end{array}$ \\
\hline Produtor 2 (P2) & 35 anos & Masc. & $\begin{array}{c}\text { Ensino superior } \\
\text { (Agrônomo) }\end{array}$ & $06^{\text {b }}$ & 650 hectares & Soja, milho e gado de corte \\
\hline Produtor 3 (P3) & 59 anos & Masc. & $\begin{array}{c}\text { Ensino superior } \\
\text { (Administrador) }\end{array}$ & 04 & 150 hectares & Soja, milho, aveia. \\
\hline Produtor 4 (P4) & 39 anos & Masc. & $\begin{array}{c}\text { Ensino superior } \\
\text { (Agrônomo) }\end{array}$ & 30 & 3.400 hectares & $\begin{array}{c}\text { Soja, milho, feijão, trigo, } \\
\text { aveia branca e aveia preta }\end{array}$ \\
\hline Produtor 5 (P5) & 36 anos & Masc. & $\begin{array}{c}\text { Ensino superior } \\
\text { (Agrônomo) }\end{array}$ & 09 & 2.000 hectares & $\begin{array}{c}\text { Soja, milho, feijão, trigo, } \\
\text { aveia e cevada }\end{array}$ \\
\hline Produtor 6 (P6) & $\begin{array}{c}24 \\
\text { anos }\end{array}$ & Masc. & $\begin{array}{c}\text { Ensino superior } \\
\text { (Agrônomo) }\end{array}$ & 08 & 920 hectares & $\begin{array}{c}\text { Soja, milho, feijão, aveia } \\
\text { branca, aveia preta e cevada }\end{array}$ \\
\hline
\end{tabular}

Nota: ${ }^{a}$ número de funcionários fixos. ${ }^{\text {b }}$ Proporção maior deve-se às necessidades de mão obra permanente na atividade pecuária Fonte: Dados da pesquisa

As entrevistas foram realizadas no mês de janeiro de 2019, e duraram em média 50 minutos cada uma. As entrevistas foram transcritas e o material foi categorizado de acordo com as 4 categorias de análises que correspondiam aos itens propostos na literatura e no roteiro da entrevista. Vale ressaltar que foi utilizado o modo de grade fechada, onde as categorias são definidas preliminarmente tendo em vista a pertinência ao objetivo da pesquisa. A categorização foi conduzida de modo a diferenciar atividades relativas ao processo de capacidade absortiva que eram realizadas pelas firmas de modo autônomo e via cooperativa. Procedeu-se desta forma buscando um contra factual que permitisse responder o problema de pesquisa. Esta análise de conteúdo foi guiada pelos preceitos da análise de co-ocorrência (Bardin, 2011).

\section{DESCRIÇÃO E ANÁLISE DOS DADOS}

Na primeira dimensão do processo de capacidade absortiva foi categorizado aquilo que era pertinente à aquisição do conhecimento. O Quadro 2 apresenta os principais trechos das entrevistas.

Todos os excertos reportados no Quadro 2 ressaltam a importância da busca por informação de modo autônomo e via cooperativa. Binotto et al. (2009) são referendados aqui. Houve mais menções à busca de informações via cooperativa, o que é demonstrado no estudo dos autores, e estas menções são bastante enfáticas se comparadas aos excertos existentes na aquisição autônoma de informações. O relato do produtor P6 sobre sua busca autônoma de informações
(Coluna B) vai ao encontro das evidências de Reagans e McEvily (2003) que mencionam que uma vasta rede pode ser importante para ganhar conhecimento externo, ou seja, um indivíduo como ampla rede de contatos e experiências pode preencher espaços a cerca de conhecimentos diversificados. Já o produtor P1, em seu relato sobre um evento realizado pela cooperativa (Coluna $\mathrm{A}$ ), enfatizou que acessa inovações graças à isso. Ainda em linha com Binotto et al. (2009) isto reforça que o ambiente cooperativo gera antecedentes propícios para incentivar a capacidade absortiva e novos conhecimentos e novas práticas na propriedade rural.

$\mathrm{Na}$ categoria assimilação do conhecimento foi categorizado aquilo que diz respeito ao início do processo interno de utilização da informação descrito por Tepic et al. (2012). Os excertos estão no Quadro 3.

Quando indagados a respeito da assimilação do conhecimento, ficou claro em todas as falas que os produtores buscam sempre acompanhar as mudanças do setor, mas que as informações precisam ser "traduzidas" para posteriormente serem utilizadas na propriedade. O relato do produtor P2 (Coluna B) corrobora com Lane e Lubatkin (1998) que constataram que as firmas convergem os seus conhecimentos aos externos transformando-os em capacidades no sentido de atender as exigências do mercado e do setor onde estão inseridas. Já o produtor P3 (Coluna A) mencionou as áreas principais em que usa a informação como pessoal, equipamentos, plantio e inserção de novas sementes. Também foi possível perceber que para ambos os modos de absorção - o autônomo e o via cooperativa - há uma tentativa de filtro das informações. Isso reforça 
Cohen e Levinthal (1990, p.128) quando se referem à capacidade de absorção como a capacidade da empresa em "reconhecer o valor da informação nova, externa, assimilá-la e aplicá-la para fins comerciais".
Na categoria transformação do conhecimento ficou constatado que os produtores possuem o hábito de discutir as tendências do mercado com empresas, parceiros e a cooperativa, conforme descrito no Quadro 4.

QUADRO 2 - Aquisição do conhecimento

\begin{tabular}{|c|c|c|}
\hline $\begin{array}{l}\text { Fonte/ } \\
\text { excerto }\end{array}$ & A. Aquisição de informações via cooperativa & B. Aquisição autônoma de informações \\
\hline $\mathrm{P} 1$ & $\begin{array}{l}\text { "O dia de campo que a cooperativa oferece é a } \\
\text { principal forma que temos em adquirir informações, } \\
\text { pois são oferecidas palestras, realização de } \\
\text { experimentos, demonstrações, validação de novas } \\
\text { tecnologias, e muitas oportunidades de negócios em } \\
\text { relação a inovação na propriedade." }\end{array}$ & $\begin{array}{l}\text { "Tenho o hábito de participar de feiras agropecuárias para } \\
\text { buscar novas informações." } \\
\text { "As empresas parceiras (fornecedores) têm as informações } \\
\text { para nos passar em relação às novas tecnologias de ponta para } \\
\text { que haja inovação no campo". }\end{array}$ \\
\hline \multirow{2}{*}{$\mathrm{P} 2$} & $\begin{array}{l}\text { "Frequento sempre o departamento técnico da } \\
\text { cooperativa para buscar informações." }\end{array}$ & $\begin{array}{l}\text { "Participação em feiras e palestras ajuda muito na obtenção de } \\
\text { informação, pois são pessoas especializadas e nessas palestras } \\
\text { e a gente encontra muitas pessoas para trocar conhecimento." }\end{array}$ \\
\hline & $\begin{array}{l}\text { "O setor comercial também me ajuda com } \\
\text { informações relativas a venda". }\end{array}$ & $\begin{array}{l}\text { "As empresas que fornecem nossos produtos nos ajuda muito } \\
\text { com diversas informações, as informações são frescas." }\end{array}$ \\
\hline \multirow[t]{2}{*}{ P3 } & $\begin{array}{l}\text { “As informações que eu recebo da cooperativa são } \\
\text { muito importantes. A informação precisa ser diária, e } \\
\text { informação é 'poder'. A participação em palestras via } \\
\text { cooperativa me ajuda muito. Os treinamentos, cursos } \\
\text { e demonstrações principalmente o dia de Campo } \\
\text { oferecido pela cooperativa é muito importante para a } \\
\text { gente obter informações. }\end{array}$ & $\begin{array}{c}\text { "Participo de conferências não só aqui da região, mas no Brasil } \\
\text { inteiro. A internet me ajuda muito com cursos treinamentos. } \\
\text { Procuro também notícias internacionais pois me ajuda a } \\
\text { entender o que está acontecendo no mundo a respeito do } \\
\text { agronegócio." }\end{array}$ \\
\hline & & $\begin{array}{l}\text { "O networking com amigos produtores é uma grande fonte de } \\
\text { informações, sempre nos encontramos em cafés da manhã" }\end{array}$ \\
\hline P4 & $\begin{array}{l}\text { "As informações que recebo é a minha base de dados } \\
\text { e a cooperativa tem grande participação quando o } \\
\text { assunto é informação." } \\
\text { "Participo do grupo do WhatsApp da cooperativa e } \\
\text { nesse grupo nós temos também os produtores rurais } \\
\text { e os técnicos que são os agrônomos da cooperativa. } \\
\text { Nesse grupo recebo informações em tempo real... } \\
\text { (informações sobre o mercado financeiro, preços dos } \\
\text { produtos, enfim uma diversidade de informações)". }\end{array}$ & $\begin{array}{c}\text { "Participo do grupo que foi criado quando eu fiz um } \\
\text { MBA (grupo de informações) onde tem agrônomos e } \\
\text { Produtores Rurais do Brasil inteiro, onde todos os dias nós } \\
\text { compartilhamos informações em tempo real[...] (sobre bolsa } \\
\text { de valores, previsão de tempo, variedades novas, híbridos, } \\
\text { máquinas novas, novas tecnologias." } \\
\text { "Também tenho grupo de parceiros da Fazenda, onde eu } \\
\text { cadastrei todas as empresas que são minhas parceiras das quais } \\
\text { eu adquiro os produtos, serviços e tecnologias." }\end{array}$ \\
\hline \multirow[b]{2}{*}{ P5 } & $\begin{array}{c}\text { "As informações que eu recebo da cooperativa ajuda } \\
\text { no planejamento para a próxima safra, pra mim } \\
\text { informação é conhecimento." }\end{array}$ & $\begin{array}{l}\text { “As empresas parceiras sempre me passam informações, } \\
\text { inclusive informações específicas e informações técnicas, até } \\
\text { mesmo quanto ao uso de novas tecnologias” }\end{array}$ \\
\hline & $\begin{array}{c}\text { "Todas as informações que recebe da cooperativa em } \\
\text { formas de treinamento, cursos e formas de palestras } \\
\text { e até mesmo o dia de campo ajuda muito a adquirir } \\
\text { informações" }\end{array}$ & $\begin{array}{c}\text { "As empresas parceiras são grandes aliadas na transmissão } \\
\text { de informações porque essas empresas estão constantemente } \\
\text { trabalhando com pesquisas" }\end{array}$ \\
\hline P6 & $\begin{array}{l}\text { "A cooperativa me ajuda muito a me manter } \\
\text { informado, considero a informação essencial." }\end{array}$ & $\begin{array}{c}\text { "Eu sempre procuro me manter informado, por meio de redes } \\
\text { de relacionamentos, networking, parceiros, outros produtores } \\
\text { rurais, e também procuro ler bastante." }\end{array}$ \\
\hline
\end{tabular}

Fonte: Excertos extraídos dos dados da pesquisa 
QUADRO 3 - Assimilação do conhecimento

\begin{tabular}{|c|c|c|}
\hline $\begin{array}{l}\text { Fonte/ } \\
\text { excerto }\end{array}$ & A. assimilação de conhecimento via cooperativa & B. assimilação de conhecimento autônoma \\
\hline $\mathrm{P} 1$ & $\begin{array}{l}\text { "Recebo muita informação da cooperativa, só que tem } \\
\text { muitas informações que precisam ser analisadas, pois } \\
\text { muitas informações que recebemos não se adapta a nossa } \\
\text { realidade e nossa região, então a gente precisa filtrar." }\end{array}$ & $\begin{array}{l}\text { "Recebo muita informação de empresas parceiras... as } \\
\text { informações precisam ser filtradas por mim e pelo meu } \\
\text { consultor que é meu agrônomo para a gente analisar } \\
\text { realmente as informações são necessárias e realmente } \\
\text { condizem com a nossa realidade e nossa região. }\end{array}$ \\
\hline $\mathrm{P} 2$ & $\begin{array}{c}\text { "Tento acompanhar todas as mudanças que ocorrem no } \\
\text { setor, como regras, regulamentos, normas, tecnologias, } \\
\text { ou seja, as exigências do mercado... sempre trazendo } \\
\text { para dentro da propriedade no sentido de estar sempre } \\
\text { atualizado" }\end{array}$ & $\begin{array}{c}\text { "Essas mudanças normalmente a gente reconhece através } \\
\text { das empresas que adquirimos os produtos como Monsanto, } \\
\text { Bayer entre outras." }\end{array}$ \\
\hline $\mathrm{P} 3$ & $\begin{array}{l}\text { "Eu tento trazer todas as informações que recebo da } \\
\text { cooperativa para dentro da minha propriedade, tanto } \\
\text { na parte de pessoal como na parte de equipamentos e } \\
\text { plantio como inserção de novas sementes...Sempre busco } \\
\text { implementar nas rotinas da fazenda." }\end{array}$ & $\begin{array}{c}\text { “A mudança de clima é constante e sempre busco } \\
\text { processar as informações para saber lidar com essas } \\
\text { mudanças para implantar na propriedade novas } \\
\text { tecnologias.” }\end{array}$ \\
\hline $\mathrm{P} 4$ & $\begin{array}{l}\text { "O mercado é muito dinâmico e as informações precisam } \\
\text { ser traduzidas, dentro da propriedade" }\end{array}$ & $\begin{array}{l}\text { "Eu leio o máximo de informações que eu consigo e } \\
\text { procuro assimilar o que é viável para a minha realidade." }\end{array}$ \\
\hline P5 & $\begin{array}{l}\text { "Todas as informações que recebo via cooperativa, eu } \\
\text { tento assimilar e verificar quais informações é possível } \\
\text { adaptar dentro da propriedade". }\end{array}$ & $\begin{array}{l}\text { "Recebo informações diárias das empresas fornecedoras... } \\
\text { mas as informações são muitas, é preciso filtrar de acordo } \\
\text { com as minhas particularidades." }\end{array}$ \\
\hline P6 & $\begin{array}{l}\text { "Procuro absorver o maior número de informações } \\
\text { principalmente as que recebo da cooperativa para poder } \\
\text { extrair as informações mais pertinentes para a realidade } \\
\text { da minha fazenda, e poder colocar em práticas nos } \\
\text { processos diárias da fazenda" }\end{array}$ & $\begin{array}{l}\text { Recebo informação diárias de muitas empresas no setor do } \\
\text { agro... mais é importante processar estas informações. }\end{array}$ \\
\hline
\end{tabular}

Fonte: Excertos extraídos dos dados da pesquisa

QUADRO 4 - Transformação do conhecimento

\begin{tabular}{|c|c|c|}
\hline $\begin{array}{l}\text { Fonte/ } \\
\text { excerto }\end{array}$ & A. Transformação de conhecimento via cooperativa & B. Transformação autônoma de conhecimento \\
\hline $\mathrm{P} 1$ & $\begin{array}{c}\text { "O Comitê tecnológico da cooperativa foi criado com o objetivo } \\
\text { de discutir as tendências do mercado, para buscar novas } \\
\text { informações e transformar em conhecimento para os produtores." } \\
\text { "O objetivo maior do comitê é associar o conhecimento } \\
\text { externo com o que já é realizado nas propriedades, a fim de } \\
\text { melhorar os processos." }\end{array}$ & $\begin{array}{l}\text { "A assistência técnica que é repassado pelas empresas } \\
\text { parceiras é uma forma de transformar o conhecimento na } \\
\text { propriedade, ou seja, tudo o que é repassado em cursos } \\
\text { e treinamentos por estas empresas, eu levo para a minha } \\
\text { propriedade, buscando melhorar o que eu já faço." }\end{array}$ \\
\hline \multirow[t]{2}{*}{$\mathrm{P} 2$} & $\begin{array}{l}\text { "Converso muito com outros associados, com os agrônomos } \\
\text { do departamento técnico da cooperativa, e com o meu } \\
\text { próprio agrônomo, sobre as tendências do mercado e novas } \\
\text { tecnologias, para identificar o que é melhor para aumentar a } \\
\text { produtividade da propriedade." }\end{array}$ & $\begin{array}{c}\text { "Discutimos muito as tendências do agronegócio com } \\
\text { os representantes das empresas, como Bayer, Monsanto, } \\
\text { Pioner entre outras." }\end{array}$ \\
\hline & $\begin{array}{c}\text { "Procuro levar meus funcionários nos treinamentos e } \\
\text { palestras oferecidas pela cooperativa para que eles consigam } \\
\text { melhorar os processos na propriedade." }\end{array}$ & $\begin{array}{l}\text { "Tento sempre implementar nas rotinas diárias da } \\
\text { fazenda os conhecimentos adquiridos" }\end{array}$ \\
\hline
\end{tabular}


QUADRO 4 - Continuação

\begin{tabular}{|c|c|c|}
\hline $\begin{array}{l}\text { Fonte/ } \\
\text { excerto }\end{array}$ & A. Transformação de conhecimento via cooperativa & B. Transformação autônoma de conhecimento \\
\hline P3 & $\begin{array}{l}\text { "No comitê tecnológico da cooperativa a gente discutir as } \\
\text { tendências do mercado, ou seja, o que está acontecendo } \\
\text { no momento da Safra e no mercado aí depois no inverno } \\
\text { normalmente a gente discute programas, outros assuntos } \\
\text { como tratamento de soja melhoramento no solo como um } \\
\text { planejamento para as próximas safras, tudo em prol de } \\
\text { melhorar a produtividade do produtor." }\end{array}$ & $\begin{array}{l}\text { "Considero importante o networking com amigos } \\
\text { produtores. Faço a roda do chimarrão constantemente } \\
\text { com meus funcionários para repassar as informações } \\
\text { que recebo, e também para receber um feedback do que } \\
\text { está acontecendo na propriedade." }\end{array}$ \\
\hline $\mathrm{P} 4$ & $\begin{array}{c}\text { "As empresas parceiras da cooperativa dão suporte para } \\
\text { transferir as informações e ajudam com treinamentos } \\
\text { técnicos." } \\
\text { "As reuniões do comitê acontecem uma vez por mês com } \\
\text { discussão de informações que sejam relevantes para o } \\
\text { setor com o objetivo de tomar decisões que resultem em } \\
\text { melhorias na produtividade dos demais associados da } \\
\text { cooperativa." }\end{array}$ & $\begin{array}{l}\text { "Toda segunda-feira eu faço uma reunião com todos os } \\
\text { funcionários da fazenda para discutir as assertividades } \\
\text { e os erros. E também para disseminar e explorar novas } \\
\text { informações, como as novas tecnologias. Decidimos os } \\
\text { acontecimentos da semana. Tenho hábito em discutir } \\
\text { as tendências no mercado referente ao agro com os } \\
\text { meus funcionários nas reuniões, ou seja, para que os } \\
\text { processos realizados na propriedade sejam adaptados se } \\
\text { necessário. Também é repassado todo o planejamento } \\
\text { estratégico e as metas a serem cumpridas. }\end{array}$ \\
\hline P5 & $\begin{array}{l}\text { "Busco sempre verificar quais informações é possível } \\
\text { adaptar dentro da minha propriedade, transformando essas } \\
\text { informações em novos processos, novas formas de trabalhar, } \\
\text { novas formas de manejo, ou seja, fazer algo diferente dentro } \\
\text { da propriedade através das novas informações" }\end{array}$ & $\begin{array}{c}\text { "Em } 2011 \text { eu tinha } 36 \text { trabalhos de pesquisas na minha } \\
\text { propriedade. Eu cedi espaço para várias empresas e } \\
\text { universidades realizarem experimentos e pesquisas. } \\
\text { Essas pesquisas me favoreciam muito até mesmo } \\
\text { porque as informações estavam frescas dentro da minha } \\
\text { propriedade." }\end{array}$ \\
\hline P6 & $\begin{array}{l}\text { "O conhecimento é transformado em discussões através de bate } \\
\text { papos, com os agrônomos da cooperativa e por meio do comitê } \\
\text { tecnológico, onde nas reuniões nós discutimos as novidades do } \\
\text { mercado, as novas tecnologias e procuramos tomar as melhores } \\
\text { decisões para ser repassada aos outros associados." }\end{array}$ & $\begin{array}{l}\text { "Também faço reuniões a cada } 30 \text { dias com os meus } \\
\text { funcionários, para transmitir as informações." }\end{array}$ \\
\hline
\end{tabular}

Fonte: Excertos extraídos dos dados de pesquisa

Todos os produtores ao serem questionados sobre a transformação do conhecimento consentem com sua importância. Tepic et al. (2012) constataram que a interação com diferentes atores se torna relevante fonte para acumular informação. Este entendimento encontra nos argumentos do produtor $\mathrm{P} 3$ e também nos fragmentos da fala do produtor P2. Já o produtor P5 (Coluna B) mencionou que transformou conhecimento por colaboração com a universidade na realização de pesquisas em sua propriedade. Wang e Ahmed (2007) ressaltaram que as empresas com maior capacidade de absorção contam com forte capacidade em aprender com parceiros. De um ponto comparativo é possível perceber que a cooperativa é algo como um "hub" de conexões para o produtor transformar conhecimento (Coluna A).

No que tange os aspectos da exploração do conhecimento, os produtores foram questionados sobre traduzir as informações para softwares e aplicativos, bem como questionados sobre o uso de tecnologias e a rentabilidade na propriedade. Grande parte dos entrevistados mencionou que a tecnologia no campo é usar as melhorar ferramentas para melhorar a produtividade, conforme especificados no Quadro 5.

Diante das narrativas, os produtores demonstraram ser imprescindível o uso de ferramentas tecnológicas para explorar o conhecimento. Para Alves (2012) a tecnologia resulta na redução nos custos de produção, na melhora da produtividade, e de forma consequente tornando a propriedade mais lucrativa e competitiva. Araújo (2008) do mesmo modo corrobora ressaltando que o avanço tecnológico é fator decisivo para aumentar a produtividade. Isto fica demonstrado no relato do produtor P5, que comprova a literatura estudada. No mesmo sentido o produtor P4 (Coluna A) também salienta que o software é responsável 
por auxiliar a explorar o conhecimento. A fala do produtor (P1) se alinha com a afirmação de Gelinski Neto (2012) que a tecnologia possui um caráter microeconômico em aumentar a produtividade e reduzir o custo médio na propriedade dessa forma melhorando a rentabilidade do produtor. Nesta categoria percebe-se também o efeito por meio das empresas parceiras, na sugestão de tecnologias como solução pertinente para a atividade. Um aspecto importante que surgiu ao longo da investigação foi que grande parte do processo de capacidade absortiva neste contexto e setor acaba se direcionando para adoção e utilização efetiva de inovações tecnológicas. Furlan et al. (2018) evidenciaram que a utilização de tecnologias e inovações no campo aumenta a produtividade e resulta em melhor desempenho nas propriedades rurais. Corroborando com a literatura, onde Lumpkin e Dess (1996) e Rhee et al. (2010) evidenciaram que a capacidade de inovação é um dos aspectos mais relevantes do processo empresarial e reflete na capacidade da empresa em participar e apoiar novas ideias, processos criativos e experimentação, que consequentemente podem resultar em novos produtos ou até mesmo processos tecnológicos. Gellynck et al. (2015) demonstrou que a capacidade absortiva aplicada ao agronegócio gera orientação para inovação.

QUADRO 5 - Exploração do conhecimento

\begin{tabular}{|c|c|c|}
\hline $\begin{array}{l}\text { Fonte/ } \\
\text { excerto }\end{array}$ & A. Exploração de conhecimento via cooperativa & B. Exploração autônoma de conhecimento \\
\hline $\mathrm{P} 1$ & $\begin{array}{l}\text { "Utilizamos na propriedade a agricultura de precisão para } \\
\text { corrigir, uniformizar, e para elevar a fertilidade do solo } \\
\text { com rotação de culturas, conquistando melhores médias } \\
\text { produtivas e índices de renda maior. [...] } \\
\text { "Também o uso de tecnologias como: mapeamento de } \\
\text { colheita, soja intacta, uso de plantadeiras com GPS. } \\
\text { Estamos sempre atentos em relação as transformações } \\
\text { tecnológicas, onde a inovação poderá resultar em melhores } \\
\text { ganhos e na redução de gastos e insumos." }\end{array}$ & $\begin{array}{l}\text { “Também faço uso da tecnologia para explorar as } \\
\text { informações recebidas [...] uma boa plantadeira com } \\
\text { GPS e monitor fornece informações pois ajuda a } \\
\text { melhorar a distribuição de sementes e de fertilizantes } \\
\text { além de evitar sobreposições de plantio, com isso tendo } \\
\text { menos gasto com fertilizantes e sementes." }\end{array}$ \\
\hline $\mathrm{P} 2$ & $\begin{array}{l}\text { "Faço uso da agricultura de precisão na minha } \\
\text { propriedade... as piores áreas que eu colho eu faço } \\
\text { reamostragem do solo para futuras correções" }\end{array}$ & $\begin{array}{l}\text { "Adquiri o software Climat FieldView, por meio da } \\
\text { empresa parceira (fornecedor) Mon santo". } \\
\text { "Ë um software monitorado pelo celular, tablete, com } \\
\text { mapeamento de plantio, onde é possível acompanhar a } \\
\text { lavoura com suporte especializado entre outras técnicas } \\
\text { através do software é possível acompanhar desde plantio } \\
\text { até a colheita, o software também analisa o desempenho } \\
\text { da área, ai é possível acompanhar as informações e } \\
\text { cruzar as informações de plantio" }\end{array}$ \\
\hline $\mathrm{P} 3$ & $\begin{array}{l}\text { "Faço uso da tecnologia na fazenda com o objetivo de } \\
\text { melhores ganhos. Depois que passei a usar as tecnologias } \\
\text { inovadoras, meu controle de gestão melhorou muito, eu } \\
\text { tinha muita perda e agora com o uso dessas inovações } \\
\text { tecnológicas minha rentabilidade melhorou." }\end{array}$ & $\begin{array}{l}\text { GPS, monitores, colheitadeiras com tecnologia de } \\
\text { ponta, manejo de plantio e outras práticas tecnológicas } \\
\text { adquiridas de parcerias externas. }\end{array}$ \\
\hline P4 & $\begin{array}{l}\text { "Eu já faço na propriedade há bastante tempo através de } \\
\text { aplicativo a coleta de amostra de solo. Já faço desde } 2002 \text {, } \\
\text { que é a coleta por precisão, ou seja, são os mapas de precisão } \\
\text { que a gente faz periodicamente a cada } 4 \text { nos na mesma área." } \\
\text { "Também tenho hábito de usar na propriedade híbridos já } \\
\text { com tecnologias inseridas, como a soja intacta, faço uso de } \\
\text { piloto automático onde a máquina anda na mesma linha, ou } \\
\text { seja, não havendo desperdícios, uso GPS, e agricultura de } \\
\text { precisão no preparo do solo" }\end{array}$ & $\begin{array}{l}\text { "O software Climat FieldView, adquirido recentemente, } \\
\text { ajuda bastante, pois é uma tecnologia que mostra a } \\
\text { população do plantio, a velocidade, a área plantada, } \\
\text { litros por hectare na pulverização e o mapa de colheita, } \\
\text { ou seja, me auxilia a obter muitas informações, além de } \\
\text { analisar o desempenho da área" }\end{array}$ \\
\hline
\end{tabular}


QUADRO 5 - Continuação

\begin{tabular}{|c|c|c|}
\hline $\begin{array}{l}\text { Fonte/ } \\
\text { excerto }\end{array}$ & A. Exploração de conhecimento via cooperativa & B. Exploração autônoma de conhecimento \\
\hline P5 & $\begin{array}{c}\text { "Faço uso de novas tecnologias, pois acredito na otimização } \\
\text { dos recursos, na diminuição dos custos e a para a eficiência } \\
\text { na produtividade." } \\
\text { "Faço uso da inoculação no suco do plantio, não é uma } \\
\text { tecnologia recente, mas tem dado muito certo na minha } \\
\text { propriedade." }\end{array}$ & $\begin{array}{c}\text { "[...] uso aplicativos no sentido de buscar informações, } \\
\text { como exemplo sobre o clima. [...] Esses tipos } \\
\text { de aplicativo ajudam na tomada de decisão e no } \\
\text { planejamento estratégico da empresa." }\end{array}$ \\
\hline P6 & $\begin{array}{l}\text { "Agricultura de precisão, soja intacta, procuro adquirir } \\
\text { híbridos com novas tecnologias, maquinários eficientes." }\end{array}$ & $\begin{array}{c}\text { "O software Climat FieldView proporciona tecnologia, } \\
\text { melhorando os processos na propriedade." }\end{array}$ \\
\hline
\end{tabular}

Fonte: Excertos extraídos dos dados da pesquisa

\section{CONSIDERAÇÕES FINAIS}

Nosso estudo buscou compreender como as cooperativas agropecuárias influenciam o processo de capacidade absortiva de produtores rurais associados. Identificamos que a busca por conhecimento externo ocorre tanto individualmente como via cooperativa, num processo colaborativo, no qual o compartilhamento de informações e experiências ocorrem rotineiramente. Essa rotina foi institucionalizada na Cooperativa com a criação de um programa denominado Comitê Tecnológico que, juntamente com o Departamento Técnico, se servem de diversos Mecanismos de Integração Social (MIS) para propiciar aos cooperados o aprimoramento da CA nas quatro dimensões: aquisição; assimilação; transformação e exploração do conhecimento externo. Nosso estudo mostra que a cooperativa prioriza inovações tecnológicas. É importante observar que os MIS utilizados se servem, também, de relações extraorganizacionais com empresas parceiras da Copercampos ${ }^{\circledR}$, confirmando o modelo de Todorova e Durisim (2007) quando argumentam que estas interações permeiam todo processo da CA.

Sobre os aspectos da assimilação do conhecimento, verificou-se que os produtores buscam sempre acompanhar as mudanças do setor, pois assimilam um grande volume de informações externas, com o propósito de absorção das informações internas, a fim de adaptá-las e aplicá-las em suas propriedades. Quanto à transformação do conhecimento, foi constatado que todos os produtores possuem o hábito de discutir as tendências do mercado com empresas parceiras, com o Departamento Técnico da cooperativa e também no Comitê Tecnológico que foi crido para discutir e filtrar de informações externas. O estudo demonstrou que a exploração do conhecimento na propriedade rural, é principalmente orientada para o uso de tecnologias, ou seja, através do uso de softwares e equipamentos. As informações geradas pelos softwares auxiliam na tomada de decisão, pois possibilita analisar o desempenho da área plantada e dessa forma é possível otimizar os recursos, com o objetivo de minimizar os custos, melhorando a produtividade e aumentando a rentabilidade. $\mathrm{Na}$ análise de co-ocorrência realizada foi possível avaliar que a cooperativa serve como um ente mediador neste processo que filtra, agiliza e facilita o processo de CA, desde a aquisição até a exploração dos conhecimentos.

Em relação a adoção de práticas de inovação tecnológicas - algo que emergiu durante o estudo - foi constatado que novas informações facilitam no processo de inovações na propriedade, bem como o uso de softwares agrícolas. A pesquisa certificou que a inovação para os produtores rurais está relacionada com uso de novas ferramentas e de novos processos, bem como o uso de novas tecnologias. Identificou-se também que a frequência do networking auxilia no processo de assimilação das informações e ajuda o processo de inovação. Por meio dos relatos, as informações recebidas por meio da tecnologia é fator determinante para a inovação na propriedade rural.

E por fim vale ressaltar a importância do papel da cooperativa no sentido de propiciar um ambiente colaborativo na troca de informações em relação aos seus associados, ou seja, os produtores rurais buscam assimilar as informações externas a fim de adaptá-las e aplicá-las em suas propriedades com o auxílio da rede de colaboração da cooperativa. A assimilação, adaptação e adoção de inovações possibilita à estas propriedades rurais melhorar sua posição competitiva.

Contudo, como limitação do estudo, destaca-se o baixo número de entrevistados, bem como as entrevistas realizadas apenas com produtores com cultivo acima de 150 hectares e possuir algum tipo de tecnologia. Se aplicado a um contingente de outros produtores (que são, numericamente, em maior número), podem ser encontrados 
resultados que talvez diferenciem do nosso estudo. Assim, como recomendação para estudos futuros, sugerimos outras aplicações empíricas com finalidades semelhantes, a um universo maior.

\section{AGRADECIMENTOS}

Agradecimento à Coopercampos ${ }^{\circledR}$ pelo acesso, e à Unoesc pela bolsa concedida à doutoranda.

\section{REFERÊNCIAS}

ALVES, E. (2012). Nosso problema de difusão de tecnologia. Ministério da Agricultura Pecuária e Abastecimento. Brasília. Revista de Política Agrícola. No 1 - Jan./Fev./Mar. 2012. Recuperado de: <http// www. ainfo.cnptia.embrapa.br/digital/.../item/.../Nossoproblema-de-difusao.pd...>

ARAÚJO, M. J. (2008). Fundamentos de Agronegócios. Atlas.

BALESTRIN,A.;VERSCHOORE, J.(2010). Aprendizagem e inovação no contexto das redes de cooperação entre pequenas e médias empresas. Organizações \& Sociedade, 17(53), 311-330.

Barcellos, J. O. J., Lampert, V. N., Grundling, R. D. P., \& Canellas, L. C. (2010). A empresa rural do século XXI no contexto do agronegócio brasileiro. Recuperado de http://www.ufrgs.br/nespro/sysdownloads/arquivos/ outros/A_EMP_RURAL_DO_SECULO_XXI.pdf.

BARDIN, L. (2011). Análise de conteúdo. Edições 70.

BINGHAM, C. B., EISENHARDT, K. M., \& FURR, N. R. (2007). What makes a process a capability? Heuristics, strategy, and effective capture of opportunities. Strategic Entrepreneurshi Journal, 1(1-2), 27-47

BINOTTO, E., SIQUEIRA, E. S., \& NAKAYAMA, M. K. (2009). Criação de conhecimento no agronegócio: estudo de casos. Revista de Administração da UFSM, 2(3), 367-384

BUAINAIN, A. M., ALVES, E., SILVEIRA, J.M., \& NAVARRO, Z. (2014). Sete teses sobre o mundo rural brasileiro. Revista de Política Agrícola, 22(2), 114-115
CHANDLER, A. D. (1992). Organizational capabilities and the economic history of the industrial enterprise. Journal of economic perspectives, 6(3), 79-100

COHEN, W. M., \& LEVINTHAL, D. A. (1989). Innovation and Learning: The Two Faces of R\&D. Economic Journal, 99(397), 569-596

COHEN, W. M., \& D. A. LEVINTHAL. (1990). Absorptive Capacity: A New Perspective on Learning and Innovation. Administrative Science Quarterly, 35(1), 128-152.

CONFEDERAÇÃO DA AGRICULTURA E PECUÁRIA DO BRASIL. (2020). Panorama do Agro. Recuperado de: https://www.cnabrasil.org.br/cna/ panorama-do-agro\#: :text $=\mathrm{As} \% 20$ estimativas $\% 20 \mathrm{e} \% 20$ proje $\% \mathrm{C} 3 \% \mathrm{~A} 7 \% \mathrm{C} 3 \% \mathrm{~B} 5$ es $\% 20$ mais, frente $\% 20 \mathrm{a} \% 20$ $2019 \% 5 \mathrm{~B} 2 \% 5 \mathrm{D}$

COOK, M. (1995). The future of U.S. Agricultural Cooperatives: A Neo-Intitucional Approach. American journal of agricultural economics, 77(5), 1153-1159

CHRISTENSEN, C., SUAREZ, F., \& UTTERBACK, J. (1998). Strategies for Survival in Fast Changing Industries. Management Science, 44(12-part-2), S207-S220.

DOSI G., NELSON, R. R., \& WINTER, S. G. (2000). Introduction: The Nature and Dynamics of Organizational Capabilities. In: Dosi, G., Nelson, R. R., \& Winter, S. G. (Eds.). The nature and dynamics of organizational capabilities. (Vol.1, pp. 1-22). Oxford: Oxford university press.

FERREIRA, J., \& FERNANDES, C. (2017). Resources and capabilities' effects on firm performance: what are they?. Journal of Knowledge Management, 21(5), 1202-1217.

FONTANARI, E. (2017). The Modern Agricultural Cooperative: A Cognitive-Knowledge-Based Approach. Euricse Working Papers, 91|17.

FULTON, M, E.; HUETH, B. (2009). Cooperative Conversions, Failures and Restructurings: An Overview. Journal of Cooperatives, 23.

FURLAN, M; ANGNES, J. S., \& MOROZINI, J. F. (2018). Capacidade absortiva em propriedades rurais de agricultores associados a uma cooperativa agroindustrial. Cadernos Ebape. br, 16, 302-317. 
GELlYNCK, X., CÁRdENAS, J., PIENIAK, Z., \& VERBEKE, W. (2015). Association between innovative entrepreneurial orientation, absorptive capacity, and farm business performance. Agribusiness, 31(1), 91-106.

Gelinski Neto, F. (2012). Tecnologia na agricultura: produtividade e renda. UFSC. Recuperado de http// fgelneto.paginas.ufsc.br/.../TEC-NA-AGRICULTURAVERSÃO-FINAL-.

HELFAT, C. E., \& PETERAF, M. A. (2009). Understanding dynamic capabilities: progress along a developmental path. Strategic Organization, 7(1), 91-102. doi: $10.1177 / 1476127008100133$

HOSKISSON, R. E., WAN, W. P., YIU, D., \& HITT, M. A. (1999). Theory and research in strategic management: Swings of a pendulum. Journal of management, 25(3), 417-456.

JANSEN, J. J., VAN DEN BOSCH, F. A., \& VOLBERDA, H. W. (2005). Managing potential and realized absorptive capacity: how do organizational antecedents matter?. Academy of management journal, 48(6), 999-1015.

KARKOULIAN, S., HARAKE, N. A., \& MESSARRA, L. C. (2010). Correlates of organizational commitment and knowledge sharing via emotional intelligence: An empirical investigation. The business Review, 15(1), 89-96.

KIM, L. (1997). The Dynamics of Samsung's Technological Learning in Semiconductors. California Management Review, 39(3), 86-100.

LANE, P. J., KOKA, B. R., \& PATHAK, S. (2006). The Reification of Absorptive Capacity: A Critical Review and Rejuvenation of the Construct. Academy of Management Review, 31(4), 833-863.

LANE, P. J., \& LUBATKIN, M. (1998). Relative Absorptive Capacity and Inter-Organizational Learning. Strategic Management Journal, 19(5), 461-477.

LUCHESI, E. S. F. (2012). Gestão do Conhecimento nas Organizações. Companhia de Engenharia de Tráfego. São Paulo. Recuperado de http://www.cetsp.com.br/ media/117897/nota\%20tecnica\%20221.pdf.

LUMPKIN, G. T., \& DESS, G. G. (1996). Clarifying the Entrepreneurial Orientation Construct and Linking it to
Performance. The Academy of Management Review, 21(1), 135-172.

MINISTÉRIO DA AGRICULTURA, PECUÁRIA E ABASTECIMENTO. (2013). Projeções do agronegócio: Brasil 2012/2013 a 2022/2023. Recuperado de http:// bibspi.planejamento.gov.br/ handle/iditem/187.

NILSSON, J.; SVENDSEN, G. L. H.; SVENDSEN, G. T. (2012). Are Large and Complex Agricultural Cooperatives Losing Their Social Capital? Agribusiness, 28(2), 187-204.

REAGANS, R., \& MCEVILY, B. (2003). Network Structure and Knowledge Transfer: The Effects of Cohesion and Range. Administrative Science Quarterly, 48(2), 240-267.

RHEE, J., PARK, T., \& LEE, D. H. (2010). Drivers of Innovativeness and Performance for Innovative SMEs in South Korea: Mediation of Learning Orientation. Technovation, 30(1), 65-75.

REZAEI-ZADEH, M., \& DARWISH, T. K. (2016). Antecedents of absorptive capacity: A new model for developing learning processes. The Learning Organization, 23(1), 77-91.

RITOSSA, C. M., BULGACOV, S. (2009). Internationalization and Diversification Strategies of Agricultural Cooperatives: a Quantitative Study of the Agricultural Cooperatives in the State of Parana. BARBrazilian Administration Review, 6(3), 187-212.

SHANAHAN, C. J., HOOKER, N. H., SPORLEDER, T. L. (2008). The Diffusion of Organic Food Products: Toward a Theory of Adoption. Agribusiness, 24(3), 369-387.

SPENDER, J. C. (1996). Making Knowledge the Basis of a Dynamic Theory of the Firm. Strategic Management Journal, 17(S2), 45-62.

SPORLEDER, T. L. (1992). Managerial economics of vertically coordinated agricultural firms. American Journal of Agricultural Economics, 74(5), 1226-1231.

SZINTOWSKI, A. M. (2017). Uma análise sobre a Capacidade Absortiva em unidades de produção de soja no Estado do Mato Grosso do Sul (Brasil) [Tese de Doutorado, Universidade do Vale do Rio dos Sinos - Unisinos]. http:// www.repositorio.jesuita.org.br/handle/UNISINOS/6669. 
SZULANSKI, G. (1996). Exploring Internal Stickiness: Impediments to the Transfer of Best Practice within the Firm. Strategic Management Journal, 17(S2), 27-43.

TEECE, D. J., PISANO, G., \& SHUEN, A. (1997). Dynamic capabilities and strategic management. Strategic management journal, 18(7), 509-533.

TEPIC, M., TRIENEKENS, J. H., HOSTE, R., \& OMTA, S. W. F. (2012). The Influence of Networking and Absorptive Capacity on the Innovativeness of Farmers in the Dutch Pork Sector. International Food and Agribusiness Management Review, 15(3), 1-34.

TIEMESSEN, I., LANE, H, W., CROSSAN, M., \& INKPEN, A. C. (1997). Knowledge Management in International Joint Ventures. In P. W. Beamish, \& J. P. Killing (Eds.), Cooperative Strategies: North American Perspective. New Lexington Press.

TODOROVA, G., DURISIN, B. (2007). Absorptive Capacity: valuing a reconceptualization. Academy of Management Review, 32(3), 774-786.

VALENTINOV, V. (2007). Why are cooperatives important in agriculture? An organizational economics perspective. Journal of Institutional Economics, 3(1), 55-69.

VAN DEN BOSCH, F., VOLBERDA, H., \& BOER, M. (1999). Coevolution of Firm Absorptive Capacity and Knowledge Environment: Organizational Forms and Combinative Capabilities. Organization Science, 10(5), 551-568.

VASCONCELOS, F. C., \& CYRINO, A. B. (2000). Vantagem competitiva: os modelos teóricos atuais e a convergência entre estratégia e teoria organizacional. Revista de Administração de Empresas, 40(4), 20-37.

VERMEIRE, B., VIAENE. J., \& GELLYNCK, X. (2009). Effect of Uncertainty on farmers decision making: case of animal manure use. Applied Studies in Agribusiness and Commerce, 3(5-6), 7-13.

VESPO, R. (2013). Using Absorptive Capacity and Knowledge Management: A case study of Swedish housing cooperatives' knowledge promoting activities [Master thesis, Södertörn University, Suécia]. http://www. diva-portal.org/smash/get/diva2:643843/FULLTEXT01.

WANG, C. L., \& AHMED, P. K. (2007). Dynamic capabilities: A review and research agenda. International Journal of Management Reviews, 9(1), 31-51.

WILSON, K., \& KEVILL, A. (2017, june). Understanding the Role of Dynamic Capabilities within Rural MicroEnterprises: The Case for the Development of a Research Agenda. In Rural Entrepreneurship Conference, Newcastle, United Kingdom. Recuperado de: https:// research.ncl.ac.uk/media/sites/researchwebsites/ ruralenterpriseuk/Wilson\%20and\%20Kevill.pdf.

PAIVA, C. M. N., \& LEME, P. H. M. V. (2018, outubro). Construção de Mercados: Uma discussão sobre a relação entre Arranjos Sociomateriais e Práticas de Mercado. In EnANPAD - Encontro Anual Da Associação Nacional Dos Programas de Pós-Graduação Em Administração, Curitiba.

ZAHRA, S. A., \& GEORGE, G. (2002). Absorptive Capacity: A Review, Reconceptualization, and Extension. Academy of Management Review, 27(2), 185-203. 